\title{
Estudo da via de acesso longitudinal para punção e cateterização da artéria digital palmar comum II no cavalo (Equus caballus) para fins arteriográficos
}

\section{A study of longitudinal approach to puncture and catheterization of a. digitalis palmaris II in the horse (Equus caballus) to perform arteriography}

\author{
Daniel Augusto Barroso Lessa ${ }^{1}$, Antônio Carlos da Silva ${ }^{2}$, Marco Antônio Leite Restum ${ }^{3}$
}

\section{Resumo}

Os autores descrevem uma via de acesso longitudinal na região metacárpica medial para punção e cateterização da artéria digital palmar comum II com finalidade arteriográfica. Utilizam 11 cavalos de equitação, sem raça definida, executando 14 acessos cirúrgicos. Após sedação, miorrelaxamento, contenção farmacológica e mecânica com o animal no decúbito lateral desejado promovem a anestesia regional. Executam o acesso entre 0 terço proximal e médio, no sulco metacárpico medial, através da divulsão do plano fascial superficial, secção do profundo e divulsão da bainha neurovascular. Discutem a técnica, suas vantagens e desvantagens, bem como suas possiveis complicações.

Palavras chave: equino; artéria; cirurgia

\section{Introdução}

Estudando as técnicas para arteriografia palmar no cavalo, observou-se que Coffman et al. (1970) executam acesso à artéria digital palmar comum II, por incisão medial da pele de $5 \mathrm{~cm}$ entre o músculo flexor digital profundo e o ligamento suspensório, ao nível da articulação metacarpofalângica. Botti e Mandrioli (1982) incisam pele e fáscia numa extensão de $2 \mathrm{~cm}$ ao nível do terço superior e médio do metacarpo. Hertsch $(1983,1985)$ incisa pele e fáscia medialmente a um palmo distal do carpo e disseca cirurgicamente a artéria digital palmar II. Silva (1988) executa incisão de 2 a $3 \mathrm{~cm}$ transversal na face medial da região metacárpica entre o terço médio e o proximal. Divulsiona a fáscia superficial, secciona a profunda, isola e repara a artéria digital palmar comum II com fio cordonet, após divulsionar a bainha neurovascular e observar o pulso. Conclui-se assim que a literatura disponível, em quase sua totalidade, se apresenta escassa quanto a informações pormenorizadas sobre as técnicas de vias de acesso, fato que dificulta o estudo quanto à execução das mesmas.

Desta forma procura-se efetuar um estudo descritivo do acesso, discutindo-se suas vantagens e desvantagens, bem como suas possíveis complicações.

\section{Material e Métodos}

Utilizaram-se 11 cavalos de equitação, com e sem histórico de claudicação, sem raça definida, com idade entre 6 e 21 anos, pesando de 300 a $450 \mathrm{~kg}$, pertencentes ao $2^{\circ}$ Regimento de Cavalaria de Guardas Andrade Neves e à Escola de Equitação do Exército, Ministério do Exército, Rio de Janeiro (Tabela). Realizaram-se 14 acessos cirúrgicos.

Obteve-se a sedação pela administração intra venosa de detomidina na dose de 0,6 a 1,2 ml. Após a instalação do efeito do fármaco (5 a $10 \mathrm{~min}$.), perfundiu-se, pela mesma via, uma solução contendo $3 \mathrm{~g}$ de tiopental sódico e $50 \mathrm{~g}$ de éter gliceril guaiacol em 1 litro de soro fisiológico (1 $\mathrm{ml} / \mathrm{kg}$ de peso corpóreo) até que o animal assumisse a posição de decúbito lateral. A solução restante foi utilizada para manutenção do estado de contenção farmacológica e sedação.

Executou-se a antissepsia do membro a ser operado e obteve-se a anestesia regional por bloqueio infiltrativo subcutâneo transversal, com $5 \mathrm{ml}$ de lidocaína a $2 \%$ sem vaso constritor, na face medial, logo abaixo da articulação carpo-metacárpica.

Estudou-se a anatomia de superfície da região metacárpica medial através da inspeção e palpação do relevo (sulcos e projeções). O sítio cirúrgico foi delimitado

\footnotetext{
1 Instituto de Veterinária, Universidade Federal Rural do Rio de Janeiro, UFRRJ, km 47 da Antiga Rodovia Rio São Paulo, 23851-970 Seropédica, RJ, Brasil

2 Instituto Biomédico, Universidade Federal Fluminense, UFF, Rua Hernani de Mello 101, 24210-130 Niterói, RJ, Brasil

${ }^{3}$ Centro Hípico do Exército, Ministério do Exército, Av. Bartolomeu de Gusmão 453, 20941-160, Rio de Janeiro, RJ, Brasil
} 
Tabela - Distribuição, identificação dos animais, tamanho da incisão de pele e respectivos membros arteriografados

\begin{tabular}{|c|c|c|c|c|c|}
\hline $\begin{array}{l}\mathrm{N}^{\circ} \text { do caso } \\
\text { operado }\end{array}$ & $\begin{array}{c}\mathrm{N}^{\circ} \text { de registro } \\
\text { e origem }\end{array}$ & $\begin{array}{l}\text { Sexo } \\
\text { (anos) }\end{array}$ & Idade & Membro & $\begin{array}{c}\text { Incisão } \\
\text { de pele } \\
\text { (cm) }\end{array}$ \\
\hline 1 & 0627/RCGd & $M$ & 21 & TDC & 4,5 \\
\hline 2 & 0046/RCGd & M & 11 & TEC & 5 \\
\hline 3 & 0265/RCGd & $M$ & 21 & TEC & 5 \\
\hline 4 & 0116/RCGd & M & 19 & TDC & 5 \\
\hline 5 & 0072/RCGd & $M$ & 14 & TEC & 4 \\
\hline 6 & 0208/RCGd & $\mathrm{F}$ & 21 & TDC & 4,5 \\
\hline 7 & 0040/RCGd & $M$ & 19 & TDC & 4 \\
\hline 8 & 0479/RCGd & $M$ & 16 & TEC & 5 \\
\hline 9 & 0112/RCGd & $M$ & 17 & TDC & 4 \\
\hline 10 & 0112/RCGd & $M$ & 17 & TENC & 6 \\
\hline 11 & 0973/Es.Eq.Ex. & $M$ & 6 & TEC & 5 \\
\hline 12 & 0973/Es.Eq.Ex. & $M$ & 6 & TDNC & 5 \\
\hline 13 & 0938/Es.Eq.Ex. & $M$ & 7 & TDC & 5 \\
\hline 14 & 0938/Es.Eq.Ex. & $M$ & 7 & TENC & 4,5 \\
\hline
\end{tabular}

RCGd: Regimento de Cavalaria de Guardas Andrade Neves; Es. Eq. Ex.: Escola de Equitação do Exército; M: masculino; F: feminino; TDC: torácico direito claudicante; TDNC: torácico direito não claudicante; TEC: torácico esquerdo claudicante; TENC: torácico esquerdo não claudicante.

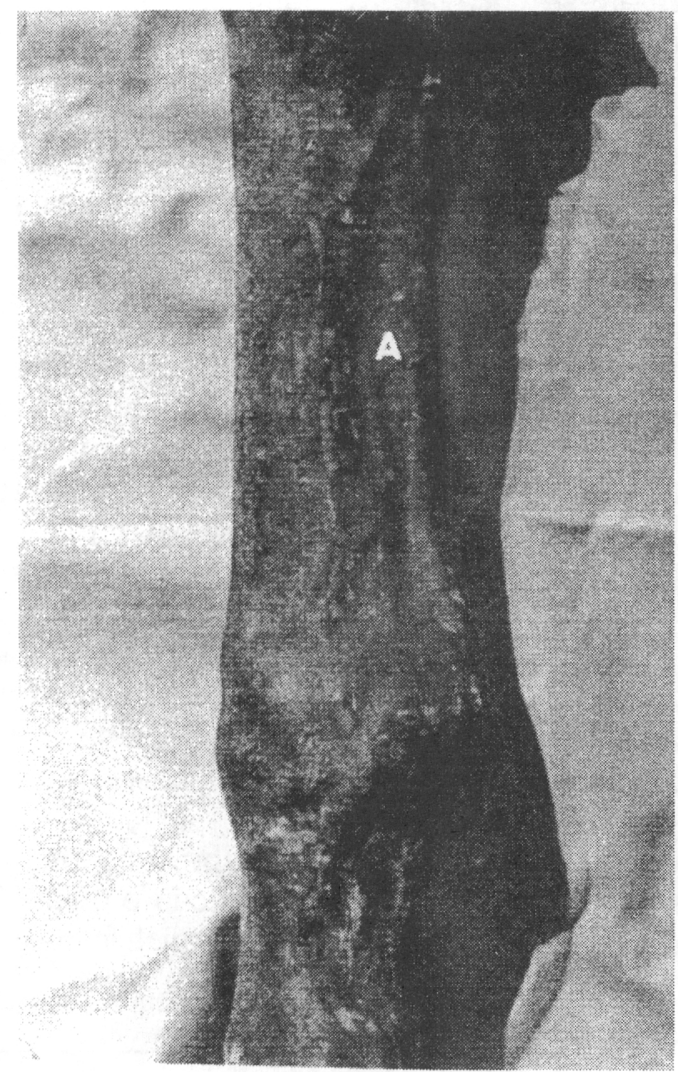

Figura 1 - Peça anatômica demonstrativa da face medial da região metacárpica e falângica proximal direita. Observação do sulco metacárpico $(\mathrm{A})$.
(Fig. 1) utilizando uma régua de aço inox. Foi medida a distância entre a tuberosidade do metacarpo e sua iminência ligamentosa medial; esta medida foi dividida em três partes iguais e o local da incisão foi delimitado entre o terço médio e o proximal, no sulco metacárpico medial, de modo a evitar traumatizar o retináculo flexor do carpo, evitar cateterizações seletivas das artérias digitais palmares próprias e para se poder estudar a artéria digital palmar comum II na maior parte de seu trajeto.

Incisou-se a pele longitudinalmente (Fig. 2) numa extensão média de $4,75 \mathrm{~cm}$ (Tabela). Prosseguiu-se com hemostasia compressiva e divulsão dos planos fasciais superficiais com tesoura de Metzembaum romba na mesma direção da incisão de pele. O plano fascial profundo foi seccionado longitudinalmente (Fig. 3). Divulsionou-se a bainha neurovascular com pinça de Kelly curva, entre o sulco formado pela veia satélite e o nervo palmar medial, afastando-os o suficiente para evidenciar a artéria digital palmar comum II de forma a permitir o acesso a esta, perceber seu pulso tanto à inspeção quanto à palpação, dar continuidade à dissecção da bainha ao redor do vaso para poder repará-la com fita cardíaca (Fig. 4) e proceder às manobras subseqüentes com vistas à punção, cateterização e arteriografia.

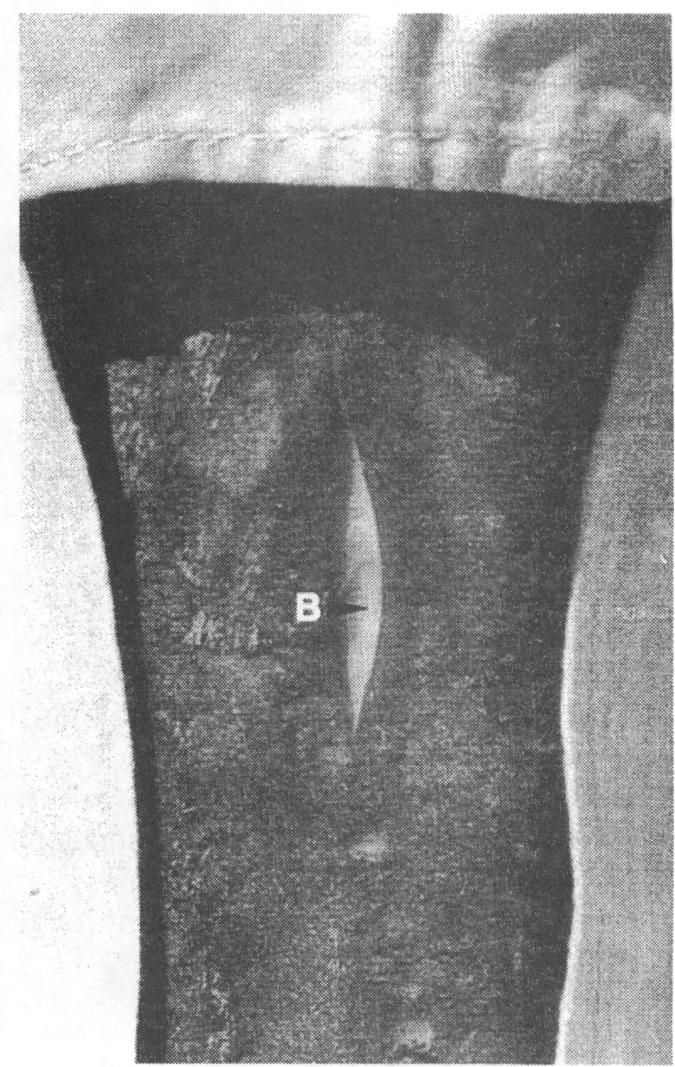

Figura 2 - Peça anatômica demonstrativa com incisão cutânea no sulco metacárpico medial na interseção do terço médio do membro torácico direito. Observação do plano fascial (B). 


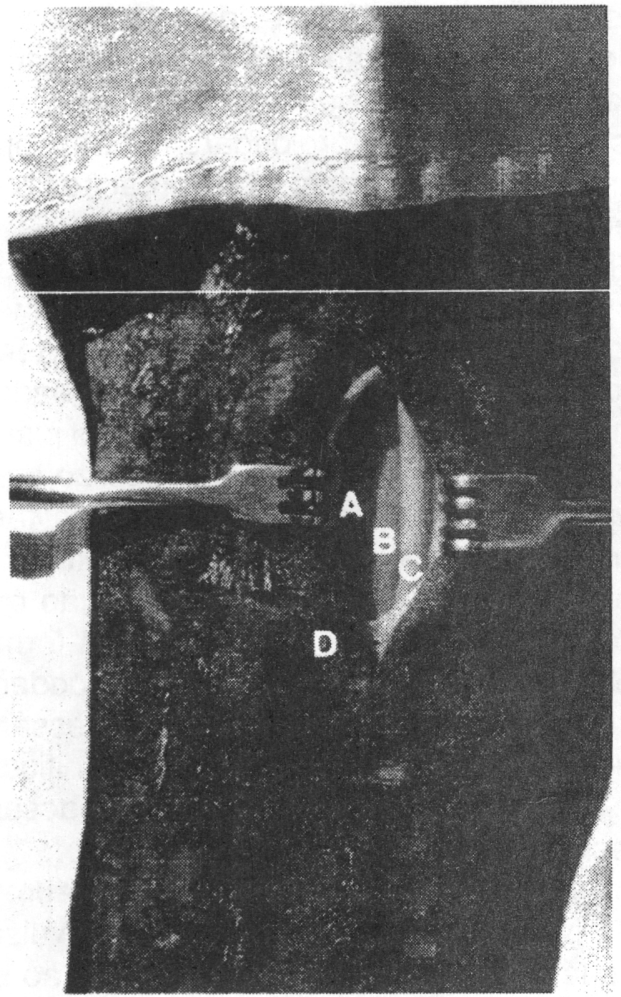

Figura 3 - Peça anatômica demonstrativa onde observa-se a veia digital (A), artéria digital palmar comum ii (B), nervo palmar medial $(C)$ e 0 plano fascial dissecado (D)

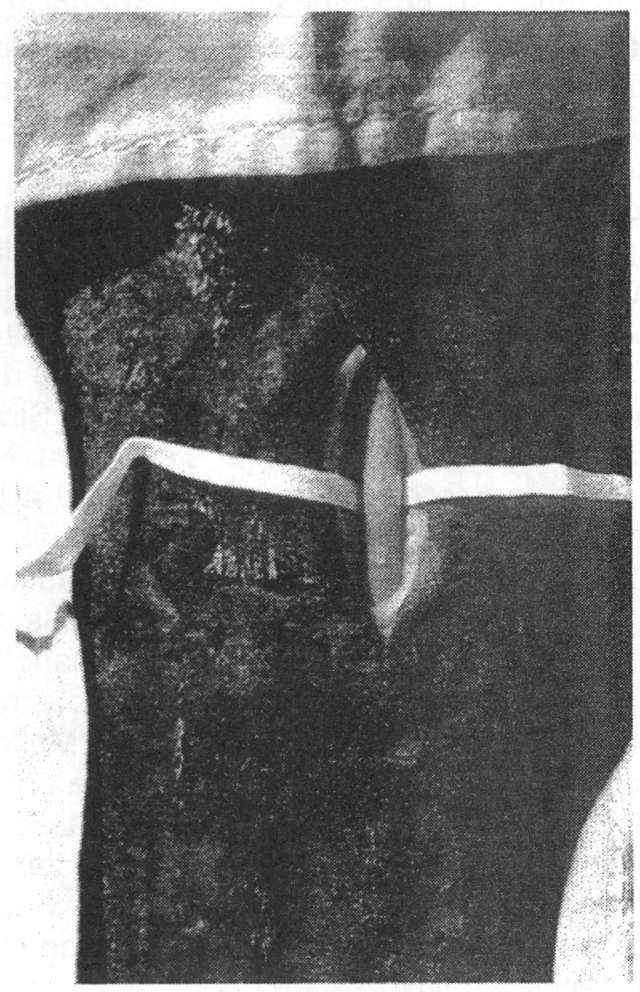

Figura 4 - Peça anatômica demonstrativa com a artéria-digital palmar comum II isolada e reparada com fita cardíaca.

\section{Resultados}

Apenas no caso 3 houve dificuldade na demarcação da área de incisão.

Nos casos 4 e 12 apareceu um ramo colateral da artéria digital palmar comum II.

No caso 12 houve tracionamento lateral intermitente do plano de pele após sua incisão.

A dissecção da bainha neurovascular circunjacente à artéria, para repará-la com fita cardíaca, facilitou as manobras de punção e cateterização.

Nos casos 3, 4 e 9 o pulso arterial não foi percebido nem à inspeção nem à palpação.

Em todos os casos, a direção longitudinal do acesso cirúrgico permitiu que durante as manobras de reparação arterial, tal estrutura não fosse deslocada acima dos limites da incisão de pele.

\section{Discussão}

Do estudo da anatomia de superfície da região metacárpica medial depende a precisão do acesso, apesar do tecido conjuntivo frouxo do plano fascial superficial permitir o deslocamento do plano de pele, de forma que a incisão cutânea fique sobre o trajeto da artéria digital palmar comum II. Acredita-se que incisões paralelas e laterais, quanto mais distantes do local de eleição, mais dificultam a exposição da artéria e desorientam o cirurgião quanto aos planos e estruturas anatômicas, o que pode acarretar dificuldades na execução do acesso e prolongar o transoperatório. $O$ animal 3 confirma os fatos acima discutidos, uma vez que superadas as dificuldades de demarcação do sítio cirúrgico, não houve dificuldade na execução do acesso.

Com relação ao local de incisão, Coffman et al. (1970), apesar de utilizarem o sulco metacárpico, o fazem ao nível da articulação metacarpofalângica. Hertsch (1973) também utiliza o sulco metacárpico medial, porém não cita em que nível, enquanto Ackerman et al. (1970) cateterizam a artéria digital palmar comun II a $5 \mathrm{~cm}$ acima da articulação metacarpofalângica em pôneis e a artéria mediana acima do carpo em cavalos. Scott et al. (1976) e Said et al. (1983) executam acesso cirúrgico na face medial do antebraço entre o músculo flexor carporadial e o rádio. Botti e Mandrioli (1982) e Silva (1988), da mesma maneira que no trabalho ora executado, elegem o terço superior e médio para o acesso cirúrgico. Hertsch $(1983,1985)$ e Litzke (1986) citam como orientação para a demarcação do local de acesso, a distância de um palmo distal ao carpo. Apesar de Silva (1988) não citar qual a unidade de comprimento que usa para medir a região metacárpica, utiliza os mesmos pontos de referência que ora são empregados, o que permite delimitar e mensurar a região metacárpica de modo que se trabalhe sempre no mesmo local, independente do indivíduo e guardadas as proporções. 
Quanto ao retináculo flexor, é uma estrutura anatômica, constituída por tecido conjuntivo fibroso, que se prolonga para a região metacárpica proximal. Dessa forma, incisões muito proximais ao carpo podem lesá-lo e provocar danos à estabilidade das articulações cárpicas bem como aos tendões relacionados a ele, haja vista o seu papel funcional.

As cateterizações seletivas podem ser provocadas quando o catéter utilizado é muito longo e/ou o ponto de punção é muito próximo às bifurcações vasculares, o que poderia acontecer se se executassem punções a partir da intersecção do terço médio com o distal. Além disso, este local permite visualizar arteriograficamente parte do terço médio do vaso e todo o segmento distal do mesmo.

Quanto à direção do acesso, apenas Silva (1988) o descreve, executando-o de forma transversal. Acreditase que, pelas características de localização profunda e trajeto longitudinal da artéria digital palmar comum II, a incisão longitudinal favoreça o ato, pois permite visualizar bem o sulco metacárpico, assim como a artéria, conferindo espaço para atuar-se de forma segura nas manobras cirúrgicas, inclusive quando ocorrem acidentes hemorrágicos como no caso 4. Além disso, parece que a dissecção longitudinal permite mobilidade suficiente ao vaso para que este seja reparado, puncionado, cateterizado e suturado com o mínimo necessário de tração para deslocá-lo do seu leito. Por outro lado, observando-se os princípios da cicatrização perprima, esta incisão parece promover pouca tensão sobre a sutura pois obedece às linhas de Langer.

No que tange às incisões, Coffman et al. (1970) e Scott et al. (1976) apesar de utilizarem o acesso em diferentes locais, empregam incisões de $5 \mathrm{~cm}$, Botti e Mandrioli (1982) fazem incisões de $2 \mathrm{~cm}$, Said et al. (1983) também utilizam incisões de $5 \mathrm{~cm}$, enquanto Silva (1988) incisa numa extensão de 2 a $3 \mathrm{~cm}$. Notam-se nos casos 1, 5, 6, 7, 9 e 14 (Tabela), onde as incisões foram menores que a média, dificuldades na dissecção da bainha neurovascular, na identificação das estruturas, na reparação, punção e cateterização, em virtude do restrito espaço criado que não permite boa visualização do sulco metacárpico e dos planos fasciais que por sua vez não permitem boa orientação anatômica para a execução do acesso e prolongam o transoperatório. Nos demais casos, onde as incisões foram maiores que a média, observa-se que o acesso ao vaso e as manobras de punção e cateterização foram mais facilmente executadas, em virtude do maior campo operatório, reduzindo-se, inclusive, o tempo cirúrgico.

Acredita-se que é importante a observação da existência de um ramo colateral da artéria digital palmar comum II no sítio cirúrgico durante a execução do acesso (dissecção fascial e da bainha neurovascular) para evitarse prolongar o transoperatório em virtude de acidentes hemorrágicos conforme o ocorrido no caso 4. Chama-se atenção para esse fato pois, apesar da dissecção cuida- dosa, a secção arterial foi favorecida pela disposição transversal deste ramo à incisão e por ficar encoberto pelo plano fascial profundo. Os acidentes hemorrágicos são indesejáveis porque além de prolongarem o transoperatório, dificultando o acesso, podem induzir reação fibroblástica e favorecer a infecção.

As dificuldades observadas na dissecção dos planos fasciais em virtude do tracionamento lateral intermitente das bordas da ferida de pele e conseqüente alteração dos planos de clivagem, parecem estar relacionadas às características do tecido conjuntivo frouxo do plano fascial superficial que permitem que a pele deslize sobre ele e apresente-se como uma trama de fibras facilmente divulsionáveis em qualquer direção. Acredita-se que este tracionamento possa desorientar o cirurgião quanto ao campo operatório, o que pode provocar a formação de novos planos de clivagem, caso prossiga, podendo levar ao aparecimento de espaço morto desnecessário e falta de precisão no acesso. Estes fatos podem dificultar o encontro e a identificação das estruturas pelo desarranjo da trama tecidual e prolongar o transoperatório.

Com relação à dissecção da bainha neurovascular, apenas Silva (1988) cita que executa sua divulsão. Acredita-se que a interposição dessa estrutura ao cirurgião, quer por dissecção incompleta ou não dissecção, pode funcionar como um obstáculo físico às manobras de reparo, punção, cateterização e síntese, dificultando e prolongando o transoperatório. Quanto à inserção do reparo, a permanência da bainha causa dificuldade para a execução da manobra e pode causar torção arterial ocasionando lesão do endotélio. Quanto à síntese, a bainha pode prejudicar a observação do orifício de punção por encobri-lo, facilitando a formação de hematomas, assim como suas conseqüências indesejáveis.

Segundo Maffei (1987) "o fenômeno de pulso arterial é correspondente ao movimento de expansão da parede arterial, determinado pela pressão pulsátil do sangue. Sendo este movimento, entretanto, dependente não só dessa pressão como também da elasticidade da parede arterial". Quanto à sua observação, apenas Silva (1988) cita que a executa para confirmar a estrutura. Neste trabalho, procurou-se observar o pulso tanto à palpação quanto à inspeção, porém, nos casos 3 e 9 não foi possível detectá-lo. Acredita-se que dentre as causas que levam a este fato, possam relacionar-se as que culminam no impedimento parcial ou total do fluxo sangüíneo naquele segmento cirurgicamente exposto: obstruçōes totais, parciais e estenoses. De acordo com Maffei (1987), "o enrigecimento por calcificação da média pode levar à não palpação do pulso e distalmente a uma oclusão ou a uma estenose importante quando o pulso estará diminuído ou mesmo ausente". Citando ainda o mesmo autor, "o fato de não se conseguir palpar um pulso pode não significar ausência de fluxo sangüíneo local, podendo verificar-se o mesmo pela utilização de um fluxômetro Doppler 
ultrassônico, mas pode indicar que a pressão do pulso está reduzida de tal forma que a expansão periódica da parede arterial seja tão discreta que não possa ser detectada pela percepção táctil dos dedos".

\section{Conclusões}

Neste trabalho, as incisões cutâneas não devem ser inferiores a $4,75 \mathrm{~cm}$ para não dificultarem os tempos cirúrgicos subseqüentes.

A dissecção longitudinal dos planos cirúrgicos foi benéfica por conferir liberdade à artéria para que esta possa ser manipulada sem que se ultrapasse os limites da incisão de pele.

\section{Abstract \\ A study of longitudinal approach to puncture and catheterization of a. digitalis palmaris II in the horse (Equus caballus) to perform arteriography}

A longitudinal approach at the metacarpal area was described being useful for arteriographical techniques which comprise puncture and catheterization of the a. digitalis palmaris II. Surgical approach - in number of 14 were made in 11 mixed bred sport horses. The lateral recumbency was possible due to sedation, muscular relaxation and mechanical and pharmacological restraint. The following procedures include regional anesthesia and incision at the medial metacarpal sulcus, between the proximal and medial areas. The complete surgical approach was possible after blunt and sharp dissection of the outer fascia and inner one, respectively, and blunt dissection of the neurovascular sheath. The technics, advantages and disadvantages were related beyond the possible complications.
Key words: equine; artery; surgery

\section{Referências bibliográficas}

Ackerman N, Garner HE, Coffman JR, Clement JW 1970. Angiographic appearance of the normal equine foot and alterations in chronic laminitis. JAm Vet Med Assoc 166(1): 58-62.

Botti P, Mandrioli R 1982. Arteriografia metacarpo-falangea e digitale nel cavallo: possibilitá di impiego clinico. Acta Med Ve 28(3): 311-318.

Coffman JR, Johnson JH, Guffy MM, Finocchio EJ 1970. Hoof circulation in equine laminitis. J Am Vet Assoc 156(1): 7683.

Herstch B 1973. Zur ateriographie der zehe der pferds. Tierarärztl. Wschr 86(24): 461-465.

Herstch B 1983. Arteriographische untersuchungen and den extremitäten bein pferd. Tese, Tierärztlichen Hochschule, Hannover, $345 \mathrm{pp}$.

Herstch B 1985. Arterielle gefässverschlüsse an der zehe als lahmheitsursache. Deutsche Veterinärmedizinische Gesellschafte 5: 143-155.

Litzke L 1986. Der einsatz der angiographie bei der lahmheitsdiagnostik. Mh Vet Med 41(10): 347-348.

Maffei FHA 1987. Diagnóstico clínico das doenças arteriais periféricas, p. 230-231. In Doenças Vasculares Periféricas MEDSI, Rio de Janeiro.

SaidAH, Khamis Y, Mahfouz MF, El-Keiley MT 1983.Angiography appearance of the metacarpus, phalanges and foot of the donkey. Zbl Vet Med 30(10): 788-795.

Scott EA, Thrall DE, Sandler GA 1976. Angiographic of equine metacarpus and phalanges: alterations with medial palmar artery and medial palmar digital artery ligation. Amer $J$ Vet Res 37(8): 869-873.

Silva AC da 1988. Arteriografia digital palmar comun II no cava10 (Equus caballus). Dissertação de Mestrado em Cirugia Veterinária, Universidade Federal Fluminense, 64 pp. 